\title{
NEVRALGIA PARATRIGEMINAL DE RAEDER COMO FORMA DE EXPRESSÃO DE ANEURISMA GIGANTE INTRACAVERNOSO
}

\author{
MARCELO GABRIEL VEGA*, ATOS ALVES DE SOUSA **, MÔNICA SCARPELLI***, \\ GERVÁSIO TELES CARDOSO CARVALHO**, ALEXANDRE AMARAL***
}

RESUMO - Os autores apresentam o caso de uma paciente do sexo feminino de 50 anos de idade com quadro de dor ocular à direita, secundária a aneurisma gigante intracavernoso diagnosticada como nevralgia paratrigeminal de Raeder. A paciente foi tratada cirurgicamente com "trapping" da carótida interna direita associado a revascularizaçāo cerebral (temporal superficial - ramo profundo da cerebral média) com ótima evolução pós-cirúrgica. Discute-se a identidade da síndrome e os diagnósticos diferenciais baseados em revisão da literatura.

PALAVRAS-CHAVE: nevralgia paratrigeminal, aneurisma carotídeo intracavernoso.

\section{Raeder paratrigeminal neuralgia as an expression of a giant intracavemous aneurysm}

SUMMARY - The authors report the case of a 50-year-old female patient with right orbital headache secondary to a giant intracavernous aneurysm, diagnosed as Raeder paratrigeminal neuralgia. The patient was surgically treated with internal carotid artery trapping together with brain revascularization (superficial temporal artery to a deep branch of the middle cerebral artery). The patient had an excellent outcome. The identity of the syndrome and the differential diagnosis are discussed based on literature review.

KEY-WORDS: paratrigeminal neuralgia, intracavernous carotid aneurysm.

A presença de dor periocular associada a manifestaçōes deficitárias simpáticas sob a forma de uma síndrome de Claude Bernard-Horner incompleta e de alteraçōes de outros nervos cranianos (III,V,VI) foi inicialmente descrita pelo neurologista norueguês George Raeder, em 1918. Seis anos após observaçāo inicial, publicou ele outros quatro caso ${ }^{15}$ delimitando, assim, uma síndrome que confere características próprias a esta entidade, denominando-a paratrigeminal por sugestāo de Monrad Krohn, devido à localizaçāo da lesāo encontrada em seu caso inicial: medial ao gânglio de Gasser, na regiāo anterior da fossa média. A partir de então sucede-se uma série de publicaçōes em que se descreve a presença dessa síndrome ${ }^{1-3,5-8,10-13,17,18}$. Devido à multiplicidade dos sintomas que formam os pilares de sustentaçāo nosológica da nevralgia paratrigeminal, podem-se observar na literatura algumas das seguintes situaçōes: em primeiro lugar, sintomatologia diferente da descrita por Raeder, seja dando ênfase a um dos sintomas, seja agregando outros sintomas; em segundo lugar, diferentes localizaçōes das lesōes causadoras da síndrome, afastando-se então o conceito "sensu stricto" de paratrigeminal.

Descrevemos o presente caso como forma de alertar acerca da possibilidade destas manifestaçōes serem provocadas por fatores potencialmente perigosos, tais como um aneurisma do

Serviço de Neurologia e Neurocirurgia da Santa Casa de Misericórdia de Belo Horizonte (SCMBH) e Disciplina de Neurocirurgia da Faculdade de Ciências Médicas de Minas Gerais(FCMMG): * Neurologista, Professor da FCMMG, Assistente da Clínica Neurológica da SCMBH; ${ }^{* *}$ Neurocirurgiāo, Professor da FCMMG, Assistente da Clínica Neurológica e Neurocirúrgica da SCMBH; *** Residente de Neurologia da FCMMG. Aceite: 15-junho-1993.

Dr. Marcelo Gabriel Vega - Rua Ceará 567, 5ªndar - 30150-310 Belo Horizonte MG - Brasil. 
seio cavernoso, não devendo este tipo de sintomatologia ser negligenciado, levando a exames complementares apropriados.

\section{RELATO DO CASO}

AS, paciente do sexo feminino, 50 anos de idade, ingressou em nosso Serviço em maio-1992 por apresentar dor ocular à direita (D) juntamente com parestesias na regiāo periocular e maxilar superior, de dois anos de evoluçāo. Seis meses após o início dos sintomas, surgiu diplopia principalmente na mirada para a D. Negava alteraçōes da acuidade visual ou outros sintomas. Relatava, claramente, dois tipos diferentes de dor: um primeiro contínuo, com componente parestésico periocular e maxilar; um segundo paroxístico, com 5 minutos a 2 horas de duraçāo e com frequência de 2 a 7 episódios semanais. Neste segundo tipo de episódio doloroso percebia clara diminuição da fenda palpebral $\mathrm{D}$, congestāo conjuntival e lacrimejamento ipsilateral, além de acentuar-se a disestesia peri e infraocular. Estes episódios foram claramente relatados pela paciente e estiveram presentes desde o início da doença. Não existia agrupamento sazonal das dores, que sempre foram unilaterais, do lado $D$, não havendo mudança de lado entre ou durante as crises e nunca ultrapassando a linha média. Era espontaneamente caracterizada como "em queimaçāo" e, quando questionada, como"pulsatil dentro do olho". Como sinais acompanhantes, sempre referindo-se à dor paroxística, observou-se: ptose palpebral, lacrimejamento e hiperemia conjuntival. A dor contínua foi catalogada como leve e suportável e a paroxística como de severa intensidade. Ambas pioravam com nervosismo e se exacerbavam no período pré-menstrual. O exame neurológico, realizado no período intercrítico, mostrou: diminuição da fenda palpebral $D$ às expensas de elevaçāo da pálpebra superior, enoftalmina $D$, hiperemia conjuntival, anisocoria (2/4) fotorreativa, paresia do VI e III nervos cranianos à $\mathrm{D}$ e reflexo corneano hipoativo à $\mathrm{D}$. Foi submetida a tomografia computadorizada e a estudo angiográfico cerebral, sendo diagnosticado aneurisma gigante de carótida interna, intracavernoso, à D (Figura 1 e 2). A paciente foi tratada cirurgicamente, tendo sido realizado simultaneamente: "trapping" de carótida interna $\mathrm{D}$ com ligadura cervical; clipagem intracraniana proximal à emergência da artéria oftálmica; e anastomose da artéria temporal superficial com ramo profundo $\left(\mathrm{M}_{2}\right)$ da artéria cerebral média. A paciente evoluiu bem no pósoperatório imediato, sem novos déficits neurológicos e com desaparecimento da dor.

Em um segundo tempo foram realizados dois estudos para melhor delimitação do quadro: avaliação oftalmológica do tipo de alteração pupilar e o teste do suor na face. O primeiro deles foi realizado (Dr. Paulo Roberto Castro e Barbosa) em ambiente com iluminaçāo escassa; as medidas iniciais das pupilas foram P.D. 2,0mm, P.E. 4,0 mm; instilaram-se 2 gotas de hidroxicloridrato de fenilefrina a 1:1000 em cada olho; passados 5 minutos repetiuse a mesma instilação; nova medida do diâmetro pupilar foi realizada 45 minutos após a primeira aplicação, verificando-se: P.D. $2,0 \mathrm{~mm}$, P.E. 7,0mm. Estes achados sāo fortemente sugestivos de pupila miótica à $D$, secundária a síndrome de Claude Bernard-Horner por lesão distal ao gânglio simpático cervical. O segundo exame, o teste de sudoraçāo da face foi realizado de acordo com as técnicas habituais, mediante aplicaçāo de tintura de iodo em toda a face que, após a secagem, foi recoberta por amido; em seguida injetou-se, no subcutâneo de pontos simétricos das regiōes frontais e maxilares, soluçāo de cloridrato de pilocarpina; o resultado mostrou preservaçāo da sudorese na hemiface $D$ o que nos permitiu inferir a preservaçāo das vias simpáticas que acompanham a carótida externa.

\section{REVISÃO ANATÕMICA DAS VIAS SIMPÁTICAS PERICAROTÍDEAS}

Sabe-se que o primeiro neurônio suprassegmentar da via simpática localiza-se na regiāo hipotalâmica posterolateral, de onde seus axônios descem através do tronco cerebral em direção ao centro cílio-espinhal de Budge, localizado na coluna intermédio-lateral $\mathrm{C}_{8}-\mathrm{T}_{2}$, onde fazem sinapse com o segundo neurônio. Os axônios deste segundo neurônio deixam a medula formando os ramos comunicantes brancos, levando os impulsos para o gânglio cervical superior, pertencente à cadeia simpática paravertebral, onde se encontram os neurônios de terceira ordem. A partir deste local, os axônios tomam vias diferentes, algumas fibras utilizam as paredes da artéria carótida externa alcançando finalmente a pele da face, onde participam dos mecanismos vasomotores e sudorais. Outro contingente de fibras, mais importante, acompanha a carótida interna e forma dois plexos: carotídeo interno e cavernoso. Destes plexos emergem fibras que utilizando o ramo oftálmico do $\mathrm{V}$ nervo craniano, dirigem-se à musculatura pupilar e ao músculo ciliar. Outras fibras, utilizando o III nervo craniano, inervam a musculatura palpebral. Desta forma pode-se compreender que lesōes de diferentes localizaçōes, como hipotálamo, mesencéfalo, bulbo, medula ou carótida, possam levar a paralisia simpática, completa ou incompleta, dando lugar a uma síndrome de Claude BernardHorner. 


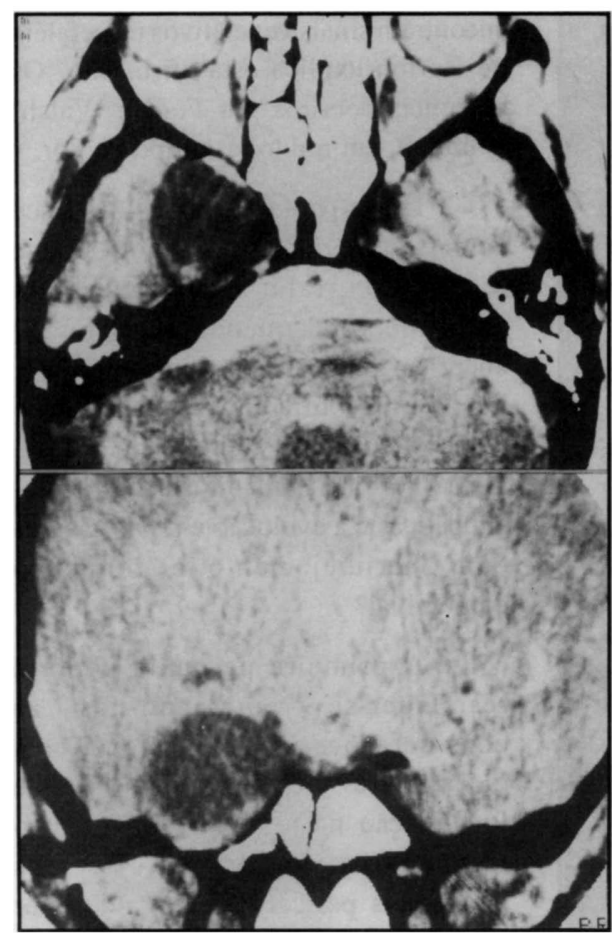

Figura 1. Caso AS. Tomografia com contraste evidenciando aneurisma gigante intracavernoso à direita.

\section{COMENTÁRIOS}

Desde a publicação dos cinco casos descritos por Raeder, a literatura mostra uma constante revisão conceitual desta síndrome. A própria classificaçäo da "International Headache Association" (1986) mostra-se reservada com respeito à identidade da neuralgia paratrigeminal, dando-lhe um exclusivo valor localizador. Vários pontos merecem uma discussāo, alguns concernentes à própria enfermidade e outros referentes à nossa paciente.

Do ponto de vista sintomatológico devese advertir que para se chegar a um diagnóstico concreto é necessário: síndrome de Claude Bernard-Horner incompleta, isto é, com preservaçāo da sudorese facial, pois as fibras que transitam pela carótida externa para inervar a face estāo íntegras; miose, enoftalmia e diminuição da fenda palpebral; dor periocular constante, $\infty \mathrm{m}$ períodos de exacerbação, quando adota características em agulhadas. Para a maioria dos autores a dor nem sempre tem as características de uma neuralgia trigeminal (vejam-se os casos do próprio Raeder). As alteraçōes podem expressar-se de formas variadas, desde simples desaparecimento do reflexo corneano até paralisia da musculatura mastigatória.

Acreditamos que um dos fatores que aportou mais confusão ao tema foi o anatômico. Quando Raeder denomina a síndrome de "paratrigeminal", o faz porque em seu primeiro caso a dor era provocada por tumor que afetava o gânglio de Gasser, comprimia o III e VI nervos cranianos em sua entrada no seio cavernoso e envolvia a carótida interna (onde, supomos, a fetava o plexo simpático pericarotídeo). Porém, nos quatro casos seguintes de Raeder, nāo podemos afirmar que o sítio da lesão era o mesmo. Os casos 3 e 5 foram causados por fratura da base do crânio, o caso 2 não tem diagnóstico definido e, finalmente, o caso 4 era de provável origem inflamatória.

Revisando a literatura, encontramos casos de síndrome de Raeder de localização muito variada, porém, sempre relacionada ao trajeto do simpático pericarotídeo. Isto implica, necessariamente, em um deslocamento conceitual no que se refere à localização e em que, como "paratrigeminal", pode-se aceitar outras localizaçōes. A síndrome de Raeder foi descrita em circunstâncias tāo diversas como: lesōes da carótida interna cervical ${ }^{3}$, carótida intrapetrosa ${ }^{5}$, carótida subcavernosa $^{14}$, carótida intracavernosa ${ }^{2,10,11}$, carótida supra-clinóide ${ }^{13}$ e fossa média ${ }^{15}$. Estes fatos nāo passaram desapercebidos para Boniuk e Schlezinger², que sugeriram a formaçāo de dois grupos: 1. Onde existe clara sintomatologia álgida, além do comprometimento de nervos para-selares (III,IV,V e VI); estes dados seriam suficientes para suspeitar-se de lesāo na fossa média e orientar a propedêutica. 2. Inclui aqueles casos sem manifestaçōes de nervos cranianos associados; é um grupo heterogêneo, em que se delineia uma superposição com as cefaléias vasculares. Este é um ponto de grande interesse conceitual, em que talvez a identidade da síndrome começa a desvanecerse. Deste modo, alguns autores ${ }^{4,16}$ a relacionam com o cluster. A grande frequência com que se 




Figura 1. Caso AS. Tomografia com contraste evidenciando aneurisma gigante intracavernoso à direita. encontram sinais vegetativos na cefaléia de Horton explica esta afirmação. Os pacientes decritos por Ford e Walsh lembram, em muito, este tipo de dor.

Outro ponto de discussāo nasce de nosso próprio caso. Interrogamos se é valido falar de neuralgia paratrigeminal quando sua causa é aneurisma gigante de seio cavernoso. Por que não foi feito inicialmente o diagnóstico com a síndrome de TolosaHunt? Por que as manifestaçōes óculosimpáticas e a evoluçảo em paroxismos com lacrimejamento e conjestāo conjuntival?

A primeira pergunta poderia responder-se remetendo-nos as consideraçōes iniciais, isto é, está claramente comprovado que a localizaçāo na fossa média é pouco frequente e que lesōes nas vias simpáticas pericarotídeas, onde quer que elas se localizem, podem levar a esta sintomatologia. $O$ diagnóstico diferencial com síndrome de TolosaHunt (obviamente que antes de se diagnosticar o aneurisma) foi realizado seguindo as orientaçōes do próprio Hunt e da "International Headache

Association": esta paciente somente apresentou sintomatologia afim nos últimos seis meses antes da realização da angiografia. Chamamos a atençāo para o tipo da dor: periocular, contínua, com episódios de exacerbação em que aparecia congestāo conjuntival e lacrimejamento. Outro ponto é o comprometimento de $\mathrm{V}_{1} \mathrm{e} \mathrm{V}_{2}$, junto à integridade do III nervo craniano nos últimos meses antes da internação.

A última pergunta responde-se de maneira mais clara. As manifestaçōes simpáticas deficitárias poderiam ser secundárias a lesāo dos plexos carotídeos interno e cavernoso, tal como acreditamos poder demonstrar através dos exames funcionais realizados. $\mathrm{A}$ integridade funcional da via simpática carotídea externa comprovada pelo teste do suor e a localizaçāo pós-ganglionar do déficit (provas pupilares) falam a favor de uma síndrome de Claude Bernard-Horner incompleta.

\section{CONCLUSÃO}

A evoluçāo da dor, suas duas etapas bem diferenciadas, uma primeira de 18 meses de evoluçāo em que prevaleciam manifestaçōes paroxísticas acompanhadas de sintomatologia simpática e do V nervo craniano e uma segunda etapa, na qual se agregam nervos cranianos tais como III e VI, sua localizaçāo e evolução, nos levaram a pensar na possibilidade de neuralgia paratrigeminal de Raeder. A realização de exames específicos mostrou-nos a presença de aneurisma gigante do seio cavernoso, causa infrequente desta síndrome. A resolução cirúrgica do aneurisma resultou em desaparecimento da dor e regressão da paralisia dos nervos cranianos. 


\section{REFERÊNCIAS}

1. Bedrossian EH. Raeder's syndrome. Arch Ophthalm 1952, 48: 620-623.

2. Boniuk M, Schlezinger NS. Raeder's paratrigeminal syndrome. Am J Ophthalm 1962, 52:1074-1084.

3. Cohen ON, Zakov ZN, Salanga VD, Dohn OF. Raeder's paratrigeminal syndrome. Am J Ophthalm 1975, 79: 1044-1049.

4. Curran RE. Raeder's syndrome. Am J Ophthalm 1975, 80: 1099.

5. Davis RH, Daroff RB, Hoyt WF. Hemicrania, oculosympathetic paresis and subcranial carotid aneurysm: Raeder's paratrigeminal syndrome (group 2). Case report. J Neurosurg 1968, 29: 91-96.

6. Ford F, Walsh FB. Raeder's paratrigeminal syndrome: a benign disorder possibly a complication of migraine. Bull Johns Hopkins Hosp 1958, 103: 296-298.

7. Grimson BS, Thompson HS. Raeder's syndrome: a clinical review . Curr Ophthalm 1980, 24: 199-210.

8. Healy JF, Zyroff J, Rosenkrante M. Raeder's syndrome associated with lesions of the internal carotid artery. Radiology 1981, 141: 101-104.

9. Headache Classification Commitee of the International Headache Society. Classification and diagnostic criteria for headache disorders, cranial neuralgias and facial pain. Cephalalgia 1988, 8 (Suppl 7).

10. Jain DC, Ahuja GK, Gonlatia RK. Intracranial internal carotid artery aneurism as cause of Raeder's paratrigeminal syndrome. Surg Neurol 1981, 16: 357-358.

11. Kashihara K, Ito H, Jamamoto S, Yamano K. Raeder's syndrome associated with intracranial internal carotid artery aneurism. Neurosurgery 1987, 20: 49-50.

12. Klingon GN, Smith WM. Raeder's paratrigeminal syndrome. Neurology 1986, 36: 750-753.

13. Law WR, Nelson ER. Internal carotid aneurysm as a cause of Raeder's paratrigeminal syndrome. Neurology 1968, 18: 43-46.

14. Nolph MD, Dion MW. Raeder's syndrome associated with internal carotid artery diletion and sinusits. Laryngocope 1982, 99: 1144-1148.

15. Raeder G. "Paratrigeminal" paralysis of oculo-pupillary sympathetic. Brain 1924, 47: 149-158.

16. Riley FC, Moyer NU. Oculosympathetic paresis associated with cluster headache. Am J Ophthalm 1971, 72: 763-768.

17. Sales M. Raeder's paratrigeminal syndrome. Arq Inst Penido Burnier 1939, 5:231-237.

18. Smith JL. Raeder's paratrigeminal syndrome. Am J Ophthalm 1958, 46: 194-201. 